\title{
Dynamics of a Circular Mindlin Plate under Mechanical Loading and Elevated Temperature
}

\author{
Anna Warminska ${ }^{1, a}$, Emil Manoach $^{2, b}$, and Jerzy Warminski ${ }^{3, c}$ \\ 1 Department of Thermodynamics, Fluid Mechanics and Aviation Propulsion Systems, \\ Lublin University of Technology, Lublin, Poland \\ 2 Institute of Mechanics, Bulgarian Academy of Sciences, Sofia, Bulgaria \\ 3 Department of Applied Mechanics, Lublin University of Technology, Lublin, Poland
}

\begin{abstract}
Dynamics of a nonlinear circular Midlin plate is studied in the paper. The mathematical model represented by partial differential equations includes nonlinear geometrical terms resulted from large displacements. The plate is subjected to mechanical and thermal loadings. The dynamics of a coupled thermo-mechanical problem is reduced from partial to ordinary differential equations. Considering the first mode reduction and uniformly distributed temperature just a single nonlinear differential equation is obtained. The bifurcation analysis shows that elevated temperature shifts the rezonanse curve and new solutions arise. Depending on initial conditions this may lead to buckling phenomenon and then relatively small oscillations around this state, symmetric periodic oscillations of large amplitude, or irregular oscillations.
\end{abstract}

\section{Introduction}

Dynamics of structures influenced by thermal field plays a very important role in the advanced and critical structural elements in mechanical or aerospace engineering [1]. The problem of thermally induced oscillations or oscillations generated by coupled mechanical and thermal loadings is crucial if the studied element is nonlinear [2]. The important coupled field analysis in a case of beam-like structural elements has been presented in [3] and [4]. Authors evidently presented that elevated temperature or heat flux may drastically change the system response. They attacked the problem solving a set of partial differential equations (PDE) using an iterative procedure based on, so called, the pseudo-normal mode method. The method gave accurate results but the computation cost was quite high and therefore rather difficult to deal with bifurcation analysis. Therefore in papers [5], [6] the reduced thermo-mechanical model of a beam has been proposed. The model allowed to study a bifurcation scenario demonstrating how the elevated temperature may change the beam response. It has been shown that among periodic also chaotic oscillations may arise and a small temperature variation may transit the system to regular motion. The bifurcation analysis based on a reduced beam model but for a nonuniform temperature distribution has been presented in [7]. The analysis performed for beams has been extended in [7] for plates. The preliminary results for circular plate model for elevated temperature and two variants external excitation frequencies have been presented. In this paper we reduce the thermo-mechanical model of the circular plate described by PDEs presented in paper [7]. The preliminary results are obtained for clamped boundary conditions.

\footnotetext{
a e-mail: a.warminska@pollub.pl

b e-mail: e.manoach@imbm.bas.bg

c e-mail: j.warminsk@pollub.pl
}

\section{Plate model}

A nonlinear model of the plate is based on Mindlin plate theory. The model proposed in this paper is extended and takes into account nonlinear displacement field, thermal and mechanical loadings. The schematic plate with indicated coordinates and dimensions is presented in Fig. 1.

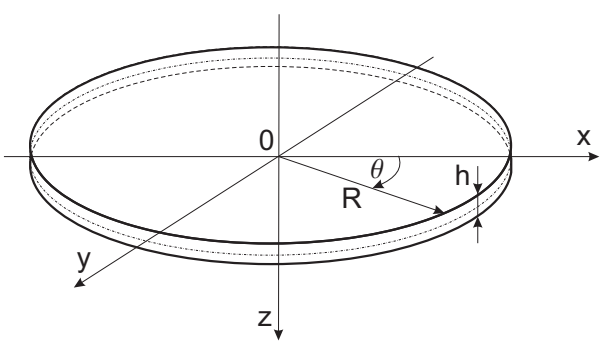

Fig. 1. Circular plate model with indicated coordinates and dimensions.

The partial differential equations of fully coupled, axissymmetric vibration of a nonlinear thermo-elastic plate is presented in [7] and has the form

$$
\begin{aligned}
& A h\left[\frac{\partial^{2} u}{\partial r^{2}}+\frac{\partial w}{\partial r} \frac{\partial^{2} w}{\partial r^{2}}+\frac{1}{r} \frac{\partial u}{\partial r}-\frac{u}{r^{2}}+\frac{1}{2 r}(1-v)\left(\frac{\partial w}{\partial r}\right)^{2}\right] \\
& =A(1+v) \alpha_{T} \frac{\partial \gamma_{T}}{\partial r} \\
& D\left[\frac{\partial^{2} \psi}{\partial r^{2}}+\frac{1}{r} \frac{\partial \psi}{\partial r}-\frac{\psi}{r^{2}}\right]-k^{2} G h\left(\frac{\partial w}{\partial r}+\psi\right)-c_{2} \frac{\partial \psi}{\partial t} \\
& -\frac{\rho h^{3}}{12} \frac{\partial^{2} \psi}{\partial t^{2}}=A(1+v) \alpha_{T} \frac{\partial K^{T}}{\partial r} \\
& k^{2} G h\left(\frac{\partial^{2} w}{\partial r^{2}}+\frac{1}{r} \frac{\partial w}{\partial r}+\frac{\partial \psi}{\partial r}+\frac{\psi}{r}\right) \\
& +A h\left[\frac{\partial u}{\partial r}+\frac{1}{2}\left(\frac{\partial w}{\partial r}\right)^{2}+\frac{v}{r} u-\frac{1+v}{h} \alpha_{T} \gamma_{T}\right]\left(\frac{\partial^{2} w}{\partial r^{2}}+\frac{1}{r} \frac{\partial w}{\partial r}\right) \\
& +A h\left(\frac{\partial^{2} u}{\partial r^{2}}+\frac{v}{r} \frac{\partial u}{\partial r}-\frac{v}{r^{2}} u+\frac{\partial w}{\partial r} \frac{\partial^{2} w}{\partial r^{2}}-\frac{1+v}{h} \alpha_{T} \frac{\partial \gamma_{T}}{\partial r}\right) \frac{\partial w}{\partial r} \\
& +c_{1} \frac{\partial w}{\partial t}-\rho h \frac{\partial^{2} w}{\partial t^{2}}=-p(r, t)
\end{aligned}
$$


where $h$ and $R$ are the plate thickness and radius, $u(r, t)$ is the in-plane displacement, $w(r, t)$ is the transverse displacement, $\psi(r, t)$ is the cross-section rotation angle, $E, G$ are Young and shear modulus, $\rho$ is material density, $\alpha_{T}$ is the coefficient of thermal expansion, and $A=\frac{E}{1-\gamma^{2}}, D=$ $\frac{E h^{3}}{12\left(1-v^{2}\right)}, \gamma^{T}(r)=\int_{-h / 2}^{h / 2} \Delta T(r, z) d z, \Delta T=T-T_{0}, T$ and $T_{0}$ are current and reference temperatures and $p(r, t)$ is external loading. We note that in Eq. 1(a) inertia term $\frac{\partial^{2} u}{\partial t^{2}}$ is neglected.

\section{Model reduction}

Taking into account that temperature is uniformly distributed along the plate radius and thickness, Eq. (1) can be simplified. Furthermore, Eq. 1(a) can be solved analytically and coordinate $u$ can be related to $w$. Thus, assuming solution in the form

$$
w(r, t)=\sum_{n=1}^{N} q_{n}(t) W_{n}(r), \quad \psi(r, t)=\sum_{n=1}^{N} q_{n}(t) \Psi_{n}(r)
$$

where $W_{n}(r), \Psi_{n}(r)$ are linear eigenmodes and $q_{n}(t)$ generalised coordinates, the PDEs can be transformed to ordinary differential equations (ODE) applying the Galerkin method. In this paper we take just one mode reduction of the clamped around plate, considering excitation distributed according to the first mode and acting around the first natural frequency. The first vibration mode is defined by Bessel functions which satisfy boundary conditions for clamped beam.

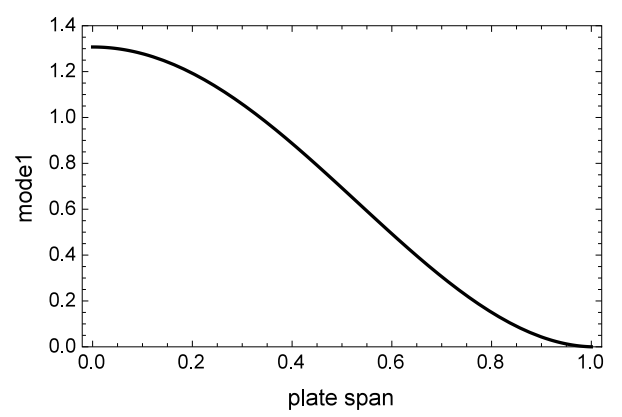

Fig. 2. First vibration mode of the clamped plate based on modified Bessel functions.

The mode shape against normalised radius is presented in Fig. 2. This mode applied in Galerkin procedure results in just one nonlinear differential equation

$\ddot{q}_{1}+2 \xi_{1} \omega_{1} \dot{q}_{1}+\omega_{1}^{2} q_{1}+F_{N L_{1}} q_{1}^{3}+F_{N T_{1}} \Delta T q_{1}=F_{N P_{1}} \sin \omega t$.

Above equation is written in the dimensionless form by introducing dimensionless time $\bar{t}=t c / R$ and displacements $\bar{u}=u / R, \bar{w}=w / R$, where $c=\sqrt{\frac{E}{\rho\left(1-v^{2}\right)}}$. The 'dot' denotes dimensionless time derivative and $q_{1}$ first dimensionless generalised coordinate. We note that in Eq. (3) $\omega_{1}$ is the first natural frequency, $F_{N L_{1}}$ coefficient occurring due to nonlinear geometrical terms, $F_{N T_{1}}$ - coefficient related to temperature variation, $F_{N P_{1}}$ - amplitude of mechanical loading and modal damping is introduced by coefficient $\zeta_{1}$.

\section{Numerical analysis}

Numerical study of the plate dynamics under mechanical and thermal loadings is performed for a clamped plate with physical parameters: $h=0.0025 \mathrm{~m}, R=0.1 \mathrm{~m}, E=0.7 \times$ $10^{11} \mathrm{~Pa}, v=0.34, \rho=2778 \mathrm{~kg} / \mathrm{m}^{3}$. This physical parameters after the modal reduction lead to dimensionless coefficients present in Eq. (3). Values of the coefficients are given in Table 1.

Table 1. Dimensionless parameters of the plate reduced model.

\begin{tabular}{lllll}
\hline parameter & $\omega_{1}$ & $c_{N L_{1}}$ & $c_{T_{1}}$ & $\zeta_{1}$ \\
\hline value & 0.07359 & 10.3516 & $-2.0033 \times 10^{-4}$ & 0.01 \\
\hline
\end{tabular}

At first a resonance curve is computed on the basis of Eq. (3) for $\Delta T=0$. The amplitude of excitation is fixed $p_{1}=4.331 \times 10^{-6}$ while frequency is varied around the first natural frequency. The influence of the geometrical nonlinear terms is demonstrated in Fig. 3 by stiffening effect present on the resonance curve. The curve has typical Duffing type nature with stable and unstable branches.

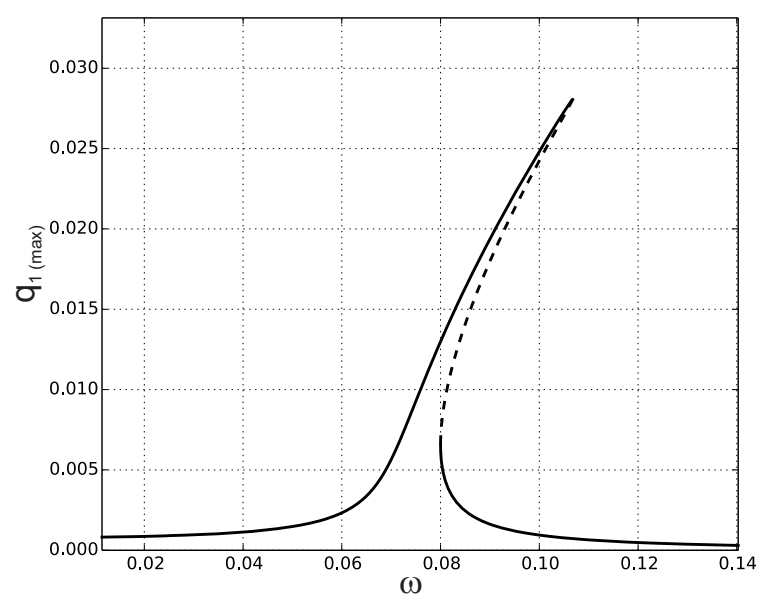

Fig. 3. Bifurcation diagram versus varied excitation frequency and fixed temperature, $\Delta T=0, p_{1}=4.331 \times 10^{-6}$.

When the temperature is elevated then we observe a shift of the resonance zone into the direction of lower frequencies. However, above a certain temperature threshold apart from the shift also qualitative changes appear. The resonance curve obtained for elevated temperature $\Delta T=$ 28 is plotted in Fig. 4. In fact, the resonance curve is shifted into lower frequency direction but furthermore, the left part of the branch becomes unstable (dashed line) and lower right branch becomes unstable too, with arising new stable solutions plotted in red. This red colour branch arises in bifurcation point leading to a buckling phenomenon. The buckling is observed by offset of the oscillation centre in a positive or negative direction.

The example of time histories is presented in Fig. 5(a) for fixed excitation frequency $\omega=0.05$. In blue and red colours the oscillations with positive or negative offset are 


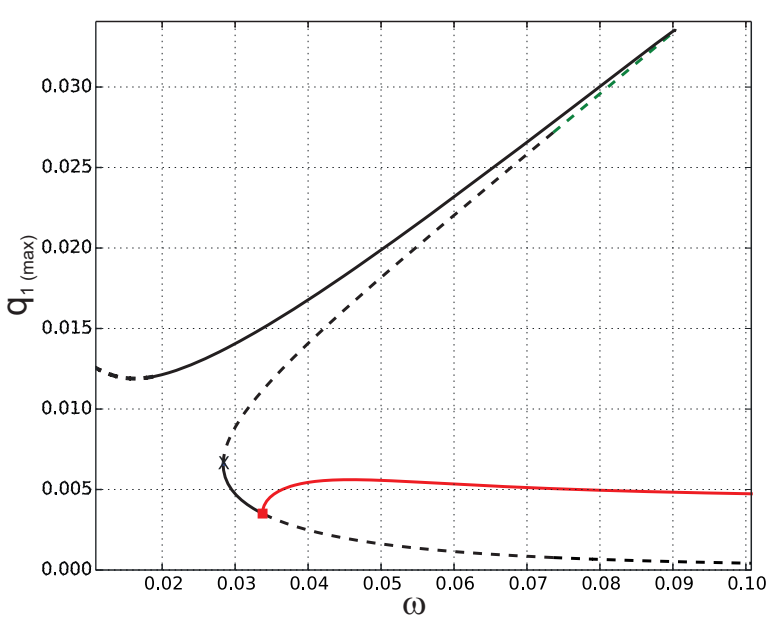

Fig. 4. Bifurcation diagram versus varied excitation frequency and fixed temperature, $\Delta T=28, p_{1}=4.331 \times 10^{-6}$.

(a)

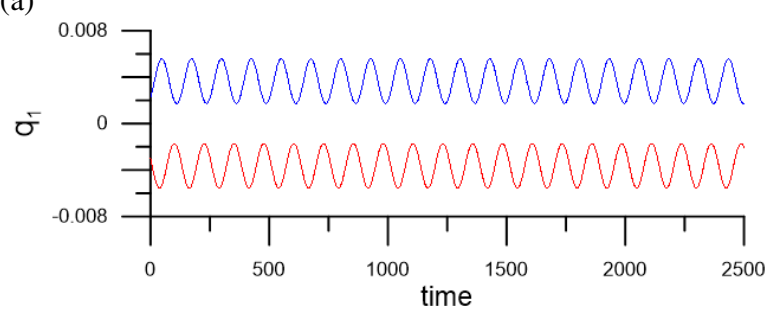

(b)

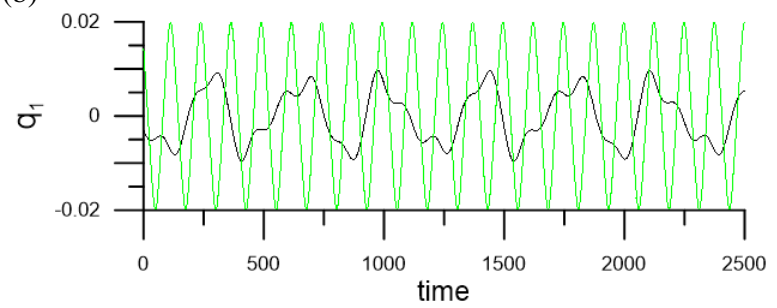

Fig. 5. Time histories for temperature $\Delta T=28$, (a) solution with positive shift (blue) and negative shift (red); (b) periodic solution (green) and irregular motion (black); $\omega=0.05, p_{1}=4.331 \times 10^{-6}$.

demonstrated, respectively. The plate can buckle in a positive or negative direction and then oscillates around that new dynamic steady state, performing relatively small oscillations.

For the same excitation frequency $\omega=0.05$ but different initial conditions the plate does not buckle but oscillates symmetrically with large amplitude equal to about 0.02 which corresponds to upper stable branch of the resonance curve (black solid line in Fig. 4). The time history of this oscillation is presented in Fig. 5(b) in a green colour. We see that in fact this solution is symmetric around zero position. The performed detailed numerical simulations for the same parameters but different initial conditions exhibited also possible irregular motion. This irregular solution is plotted in Fig. 4(b) by black line.

\section{Conclusions}

The extended model of Mindlin plate which takes into account geometrical nonlinear terms due to large deformation and influence of thermal field are presented in the paper. It is shown that the complex model, represented by partial differential equations can be reduced to ordinary differential equations taking into account a selected number of modes. The computations based on the first mode reduction show the stiffening effect of the resonance curve. The elevated temperature shifts the resonance curve into lower frequencies direction. Moreover, due to the temperature increase above a certain threshold the resonance curve changes qualitatively. For example, for temperature increase up to $\Delta T=28$, some parts of the curve become unstable and a new stable branch arises. The new solutions correspond to buckling phenomenon and periodic oscillations around this state. These small amplitude oscillations are characterised by a positive or negative offset. But for the same parameters and varied initial conditions the response can be periodic with no offset or irregular. The detailed bifurcation analysis and detection of the transition to irregular (chaotic) motion is planed to be studied in future investigations.

\section{Acknowledgements}

The first author would like to acknowledge the support received from the Polish NSF Grant S-38/M/2015, the second author the partial support from Bulgarian NSF Grant DUNK-01/3 and H2020 Marie Sklodowska-Curie ITN under grant agreement No 643095, and the third author the support from Polish NSF grant S-3/M/2015.

\section{References}

1. E.A. Thorton, Thermal structures for aerospace applications (AIAA Education Series, 1996) 1-479.

2. M. Amabili, S. Carra, J. Sound and Vibration, 321, (2009) 936-954.

3. E. Manoach, P. Ribeiro, Int. J. Mech. Sci., 46, (2004) 1589-1606.

4. P. Ribeiro, E. Manoach, J. Sound and Vibration, 285, (2005) 1093-1107.

5. A. Warminska, E. Manoach, J. Warminski, Meccanica, 49, (2014) 1775-1793.

6. A. Warminska, E. Manoach, J. Warminski, S. Samborski, Continuum Mechanics and Thermodynamics, (2014), DOI 10.1007/s00161-014-0381-6.

7. A. Warminska, E. Manoach, J. Warminski, Proceedings of ICoEV 2015 Int. Conf. on Engineering Vibration, Ljubljana, Slovenia, (2015) 1057-1066. 